This item was submitted to Loughborough's Research Repository by the author.

Items in Figshare are protected by copyright, with all rights reserved, unless otherwise indicated.

\title{
Physical activity attenuates metabolic risk of adolescents with overweight or obesity: the ICAD multi-country study
}

PLEASE CITE THE PUBLISHED VERSION

https://doi.org/10.1038/s41366-020-0521-y

PUBLISHER

Springer Nature

VERSION

AM (Accepted Manuscript)

\section{PUBLISHER STATEMENT}

This paper was accepted for publication in the journal International Journal of Obesity and the definitive published version is available at https://doi.org/10.1038/s41366-020-0521-y.

\section{LICENCE}

All Rights Reserved

\section{REPOSITORY RECORD}

Werneck, Andre O, Danilo R Silva, Adewale L Oyeyemi, Romulo A Fernandes, Jostein Steene-Johannesen, Lars Bo Andersen, Kate Northstone, et al.. 2020. "Physical Activity Attenuates Metabolic Risk of Adolescents with Overweight or Obesity: The ICAD Multi-country Study". Loughborough University. https://hdl.handle.net/2134/13241543.v1. 
Physical activity attenuates metabolic risk of adolescents with overweight or obesity: The ICAD Multi-country Study

Running title: Physical activity, obesity and metabolic risk

André O Werneck ${ }^{1}$; Danilo R Silva ${ }^{2}$, Adewale L Oyeyemi ${ }^{3}$, Rômulo A Fernandes ${ }^{1}$, Jostein Steene-Johannesen ${ }^{4}$, Lars Bo Andersen ${ }^{5}$, Kate Northstone ${ }^{6}$, Luís B Sardinha ${ }^{7}$, for the International Children's Accelerometry Database (ICAD) Collaborators

1. Laboratory of InVestigation in Exercise - LIVE. Department of Physical Education. São Paulo State University (UNESP), Presidente Prudente, Brazil.

2. Department of Physical Education, Federal University of Sergipe - UFS, São Cristóvão, Brazil.

3. Department of Physiotherapy, College of Medical Sciences, University of Maiduguri, Borno State, Nigeria.

4. Norwegian School of Sport Science, Oslo, Norway.

5.Western Norway University of Applied Sciences, Sogndal, Norway.

6. Department of Population Health Sciences, Bristol Medical School, University of Bristol, UK.

7. Exercise and Health Laboratory, Faculty of Human Kinetics, CIPER - Universidade de Lisboa, Portugal.

\section{Corresponding author:}

André de Oliveira Werneck. Department of Physical Education. São Paulo State University (UNESP), Presidente Prudente, Brazil. R. Roberto Símonsen, 300 - Centro Educacional, Pres. Prudente - SP, 19060-900. e-mail: andreowerneck@gmail.com 
49

50

51

52

53

54

55

56

57

58

59

60

61

62

63

64

65

66

67

68

69

70

71

72

73

74

75

\section{ABSTRACT}

Background: Although the benefits of physical activity (PA) at an early age are well established, there is no robust evidence of the role of PA as well as its intensities in attenuating the association between weight status and metabolic risk among adolescents. In this investigation, we analyzed the association between weight status, intensities of PA, and metabolic risk among adolescents.

Methods: Data from six cross-sectional studies in the International Children's Accelerometry Database were used $(\mathrm{N}=5,216$ adolescents; boys $14.6 \pm 2.1 \mathrm{y}$ and girls $14.7 \pm 2.0 \mathrm{y}$ ). Weight status was assessed and classified according to body mass index. Fasting glucose, triglycerides, inverse high-density lipoprotein cholesterol, and blood pressure composed the metabolic risk indicator (z-score). PA was measured by accelerometers. The estimated age of peak height velocity was used as a covariate for somatic maturation.

Results: We observed that increase in weight status showed a strong positive relationship with metabolic risk. However, adolescents with overweight or obesity in the highest tertile of PA (moderate-to-vigorous and vigorous intensity) showed a similar metabolic risk score as the normal weight groups. Moderate intensity PA seemed related to metabolic risk even within some categories of vigorous PA.

Conclusions: We conclude that PA attenuates the metabolic risk of adolescents with overweight or obesity. Although this attenuation is largely explained by vigorous PA, moderate intensity seems also important for better metabolic profile.

Key words: exercise; ICAD; metabolic syndrome; cardiovascular risk 


\section{INTRODUCTION}

The childhood obesity pandemic has changed the profile of chronic disease among children and adolescents [1]. There is increasing attention on the assessment of metabolic risk factors such as homeostatic model assessment of insulin resistance (HOMA-IR), blood pressure, fasting glucose, triglycerides and high-density-lipoprotein cholesterol (HDL-C) during early ages [2,3]. In a comprehensive sample of 4,581 participants of the International Children's Accelerometry Database (ICAD), Kuzik et al. [2] found that $45 \%$ of children and adolescents presented with at least one of these metabolic risk factors. Thus, both the prevention and treatment of early risk factors in children and adolescents should be considered as priorities.

Physical activity (PA) is well established as a key determinant in the prevention and treatment of childhood obesity and early metabolic risk factors [2,4]. However, unlike in adults $[5,6]$, the protective effect of PA in attenuating the association between weight status and metabolic risk indicators in youth is unclear. Although PA seems to have a positive influence on inflammatory markers associated with overweight and obesity during adolescence [7], little robust evidence is available in this context. Among the gaps, few studies adopted objective measures of PA, limiting their ability to assess the potential effects of different intensities of PA on metabolic health [2]. Also, maturation plays an important role on metabolic profile especially during adolescence and not accounting for its influence on metabolic markers can confound data interpretation $[8,9]$.

Identifying the role of PA and its specific intensities on metabolic risk indicators for normal weight, but especially for children and adolescents with overweight or obesity, can provide information for future international recommendations and for clinical practice regarding the prevention and treatment of early metabolic risk factors 
102 in early life. Thus, we analyzed the association between weight status, intensities of PA, 103 and metabolic risk among adolescents, with special consideration to the role of PA in 104 attenuating the positive association between obesity and metabolic risk among 105 adolescents.

107 METHODS

108

109 Design

110 The International Children's Accelerometry Database (ICAD) (http://www.mrc111 epid.cam.ac.uk/research/studies/icad) has the aim of pooling data on both cross112 sectional and longitudinal PA studies conducted among children and adolescents

113 worldwide. More information about the study process has been previously described 114 elsewhere [10]. Briefly, the dataset has pooled objectively measured ActiGraph 115 accelerometer data (ActiGraph, LLC, Pensacola, Florida). This data set used 116 standardized data reduction techniques on 46,131 raw ActiGraph data files between 1172008 and 2010 [10]. Moreover, data of sociodemographic, anthropometric, and 118 cardiometabolic factors were also pooled when available. Participants' age ranged from 1193 to 18 years. For the present study, we used data from six cross-sectional studies from 120 five countries: the ALSPAC (England), the EYHS Denmark (Denmark), the EYHS 121 Estonia (Estonia), the EYHS Portugal (Portugal), the NHANES 2003-04 (United States 122 of America), and the NHANES 2005-06 (United States of America).

124 Sample

125 The initial sample was composed of 21,667 adolescents with complete accelerometer 126 data: the ALSPAC $(n=12,746)$, the EYHS Denmark $(n=2,045)$, the EYHS Estonia 
$127(n=660)$, the EYHS Portugal $(n=1,356)$, the NHANES 2003-04 $(n=2,372)$, and the

128 NHANES 2005-06 $(\mathrm{n}=2,488)$. Due to missing data on sociodemographic, 129 anthropometry, metabolic variables, as well as invalid accelerometer data, the final 130 (included) sample was composed of 5,216 adolescents (2,730 girls): the ALSPAC $131(n=1,588)$, the EYHS Denmark $(n=1,452)$, the EYHS Estonia $(n=421)$, the EYHS 132 Portugal $(n=596)$, the NHANES 2003-04 $(n=602)$, and the NHANES 2005-06 $(n=557)$, 133 with age ranging from 10 to 17 years of age. Ethical approval for all studies were 134 obtained from the local ethics committees, including the ALSPAC Ethics and Law 135 Committee and the Local Research Ethics Committees.

\section{Metabolic risk score}

138 Fasting blood glucose, triglycerides, and high-density lipoprotein cholesterol (HDL-C) 139 were measured following a fasting period of at least 12 hours using standard clinical 140 procedures previously described, with limited between-study variation $[10,11]$. Systolic 141 and diastolic blood pressure were measured using manual and automatic methods. As 142 outcome, we created a continuous metabolic risk score, in which, z-scores from the 143 above-mentioned metabolic variables were created according to sex and chronological 144 age (year) [12]. This indicator is widely accepted as an indicator of cardiovascular risk 145 among adolescents [13]. After this, all indicators were summed to create the indicator of 146 metabolic risk score, given that HDL-C was multiplied per -1 (inverse) and mean of

147 blood pressure $\mathrm{z}$-scores $\left(\mathrm{SBP}_{\mathrm{z} \text {-score }}+\mathrm{DBP}_{\mathrm{z} \text {-score }} / 2\right)$ was included in the sum as shown in 148 the following equation:

150 Metabolic risk score: $\left(\right.$ HDL-C $\left._{\mathrm{z} \text {-score }}{ }^{*}-1\right)+$ triglycerides $_{\mathrm{z} \text {-score }}+$ fasting glucose $\mathrm{z}_{\text {-score }}+$ $151\left(\mathrm{SBP}_{\mathrm{z} \text {-score }}+\mathrm{DBP}_{\mathrm{z} \text {-score }} / 2\right)$ 
Waist circumference was not included in the overall indicator of metabolic risk

154

155

156

157 Body mass index

158 Body mass index (BMI) was used to indicate weight status, using values of stature 159

160

161

162

163

164

165

166

167

168

169

170

171

172

173

174

175

176

due to its close association with BMI, which could confound the results. A detailed description of these procedures has been described previously [10,11].

(expressed as $\mathrm{m}$ ) and body mass (expressed as $\mathrm{kg}$ ). The values provided by Cole et al. [14] were used to classify the participants as normal weight, overweight or obese, which include specific cutoff points for each age group and sex.

\section{Physical activity (PA)}

Physical activity was collected using different Actigraph accelerometers. The ALSPAC study used the Actigraph models 7164, 71256, GT1M; the EYHS Denmark, Estonia and Portugal study used the Actigraph model 7164; and the NHANES 2003-04 as well as the 2005-06 studies used the Actigraph model GT1M. All studies adopted the placement at the hip protocol. Moreover, the ALSPAC and both NHANES cohorts adopted a protocol of 7 consecutive days of wearing the accelerometer, while the EYHS Denmark, Estonia and Portugal studies adopted the 4 consecutive days ( 2 weekdays and 2 weekend days) wearing protocol. Aiming to standardize the procedures, ICAD reanalyzed PA data from accelerometers using 60 second epochs. Non-wear time was considered as 60 minutes of consecutive zeros with tolerance of 2 minutes of nonzero epochs. Moreover, aiming to minimize the missing data, we adopted the cutoff point of 500 minutes per day as a valid day of measurement and a minimum of one valid day per week. To classify sedentary time and intensities of PA (moderate [MPA], vigorous 
177 [VPA], and moderate-to-vigorous [MVPA]), we used the cut-points provided by

178 Evenson et al.[15]. A detailed description of how PA measures were pooled has been 179 described previously [10].

180

181 Confounders

182 Chronological age, peak height velocity, cohort, accelerometer wear time and mothers' 183 educational status were adopted as confounders. The age of peak height velocity was 184 estimated using a logarithm that included chronological age and stature provided by Moore et al. [16].

187 Statistical procedures

188 Characteristics of the sample were described using means and $95 \%$ confidence 189 intervals, which were used to compare characteristics between groups [17]. Linear regression models adjusted by accelerometer wear time, sedentary time, study, chronological age and age at peak height velocity were used to analyze the independent associations between intensities of PA and BMI in predicting metabolic risk. The joint association of weight status and tertiles of PA intensities in predicting metabolic risk score was analyzed by ANCOVA using confidence intervals for differences between groups. All analyzes were conducted in STATA 15.1, adopting statistical significance as $\mathrm{p}<0.05$.

\section{$198 \quad$ RESULTS}

199 From the initial sample $(\mathrm{n}=21,667)$, only 5,216 adolescents presented blood variables 200 from six different studies: the ALSPAC $(n=1,588)$, the EYHS Denmark $(n=1,452)$, the 201 EYHS Estonia ( $\mathrm{n}=421)$, the EYHS Portugal $(n=596)$, the NHANES 2003-04 $(n=602)$, 
202

203

204

205

206

207

208

209

210

211

212

213

214

215

216

217

218

219

220

221

222

223

224

225

226

and the NHANES 2005-06 $(n=557)$. Characteristics of the included and excluded sample are presented on Table 1. Included participants were relatively older, presented lower physical activity levels and higher sedentary time. However, the proportion of sex, weight status and mother's education were similar between the included and excluded sample.

Characteristics of the included sample are presented in Table 2. Girls presented lower PA levels, younger age of peak height velocity, smaller waist circumference, and lower fasting glucose and systolic blood pressure compared to boys $(p<0.05)$. On the other hand, girls had higher BMI, diastolic blood pressure, HDL-C and triglycerides $(\mathrm{p}<0.05)$.

**Table 1 about here**

Joint associations between PA intensities (VPA and MVPA) and BMI in predicting metabolic risk score are presented in Table 3. Among the models, both PA and BMI were associated with metabolic risk score. The association between VPA and metabolic risk as well as MVPA and metabolic risk were similar in both sexes. However, the inclusion of PA in the models did not contribute much to the variance in metabolic risk score already explained by BMI.

**Table 2 about here** Metabolic risk score according to tertiles of PA (MVPA and VPA) and BMI status are presented in Figure 1. The group of participants with obesity in the first 
227 tertile of both MVPA and VPA presented the most adverse values of metabolic risk.

228 Boys with normal weight in the first tertile of MVPA showed higher metabolic risk

229

230

231

232

233

234

235

236

237

238

239

240

241

242

243

244

245

246

247 Our main finding was that a lower metabolic risk in adolescents with overweight and

248 obese in the highest tertiles of PA was similar to the metabolic risk of their counterparts

249

250

251 compared to their counterparts of the third tertile. The gradual association between mutually exclusive categories of PA/weight status and metabolic risk was clearer for boys, however the third tertile of PA appeared to be a protective factor in both sexes regardless of weight status. Moreover, girls with overweight in the second tertile of PA showed similar metabolic risk of their counterparts with normal weight.

**Figure 1 about here** according to weight status and tertiles of VPA is presented in Table 4. Body mass index was significantly associated with metabolic risk score among all weight status and tertiles of VPA. MPA was associated with metabolic risk in the tertiles one and three of VPA among boys, as well as in the intermediary tertile among girls.

$$
\text { **Table } 3 \text { about here** }
$$

\section{DISCUSSION} with normal weight. We also observed that VPA seemed to be strongly associated with this modelling effect. Moderate PA also explained variations in metabolic risk, reinforcing international guidelines, which suggest $150 \mathrm{~min} /$ week of MVPA for health. 
252 To our knowledge, this is the first study that analyzed this association and the plausible

253 attenuation effect of PA on metabolic risk using a comprehensive sample with device254 based monitoring of PA.

255 Overweight and obesity are associated with several negative health outcomes 256 even among adolescents, including cardiovascular diseases [2,18]. Several mechanisms 257 may explain the positive association observed between BMI and metabolic risk. Excess 258 adipose tissue, that often goes along with increased BMI, results in the increased 259 secretion of free fatty acids [19], reduction of adiponectin [20], an increase of inflammatory markers such as IL-6 and CRP [7], and an increase in insulin resistance, all of which contribute to the hyperlipidemia, hypertension, and glucose intolerance 262 components of the metabolic syndrome [21]. PA, on the contrary, is associated with a 263 reduced metabolic risk [11] through the reduction of inflammatory factors [7] and 264 reduction of adiposity [22].

Our results suggest that, overweight/obesity are associated with metabolic risk among adolescents, but PA is capable to attenuate this association. Adolescents with 267 overweight and obesity, in the highest tertile of PA showed reduced metabolic risk 268 compared to counterparts with normal weight. A previous investigation from the ICAD 269 database found that MVPA was only positively associated with metabolic risk (presence of at least one metabolic risk factor) among children and adolescents with normal 271 weight [2], which is somewhat distinct from our findings. This difference may be 272 explained by differences in the analytical procedures used (categorical vs continuous 273 outcomes) and by differences in the age range of participants included (children + 274 adolescents vs adolescents only). In addition, we opted to adjust the analyses for 275 biological maturation, which is an important factor to be considered for both PA and 
cardiovascular risk in this age group (adolescence) [8,9]. Thus, it is possible that PA provides more benefit for groups with overweight and obese with advancing age.

The observed risk attenuation by PA was clearer for boys. This result can be explained in part by the lower level of general PA [23] and VPA among girls with overweight or obese compared to boys, potentially due to the effects of obesity on motor competence and difficulty with movement of their higher body mass [24,25]. Here, for example, girls in the third tertile showed significantly less MVPA (girls: 67 $\mathrm{min} /$ day vs boys: $\sim 94 \mathrm{~min} /$ day) and VPA (girls: $\sim 23 \mathrm{~min} /$ day vs boys: $\sim 36 \mathrm{~min} /$ day) than boys. This also suggests that higher amounts of PA are needed for metabolic risk attenuation among adolescents with overweight and obesity.

Interestingly, we observed similar results when we compared models with MVPA and VPA. Although there is clear evidence that more intense PA provides additional benefits for health [26], our findings suggests that even MPA seems important for a better metabolic profile. This may have a special practical implication, as adolescents, especially those who are overweight and obese, are more likely to take part in and adhere to PA programs of lighter intensity (i.e. MPA instead of VPA) [27].

The potential attenuation effect of PA in the association between weight status and metabolic risk can occur through different mechanisms. Both PA and obesity present convergent mechanisms to metabolic risk factors such as inflammation [7]. PA is associated with a reduction of inflammatory factors, while obesity is associated with increased inflammatory factors. Moreover, PA improves adiponectin levels while obesity decreases them $[20,28]$. In this sense, PA should be promoted for the prevention of cardiovascular diseases, even without the reduction of body weight.

Some limitations of the current study should be mentioned. First, we considered a minimum of one day of valid wear time in the present study, which can present bias as 
301 one day may not be representative of usual habitual PA. Second, the different models of 302 accelerometers used in each study could represent possible variation between study 303 outcomes. Third, the large number of missing data on metabolic variables must be 304 highlighted, being a potential selection bias for the included sample. The main 305 difference between the included and excluded sample was on chronological age, 306 physical activity level and sedentary time. However, age-adjusted estimates such as

307 BMI and mother educational status (a socioeconomic proxy) were not different between 308 the included and excluded sample. Fourth, the use of a continuous outcome has 309 operational advantages, but it is also relevant to recognize that approaches adopting a 310 categorical diagnosis of metabolic syndrome have its advantages as well. Finally, the

311 lack of control of other potential metabolic risk determinants (e.g. energy intake), and

312 the cross-sectional design preclude evidence of causality from the interpretation of the 313 findings. However, the comprehensive sample with device-based monitoring of PA

314 from four different countries represent the main strength of the study. The 315 understanding of the potential effects of different amounts and intensities of PA on 316 health is only possible through objective/device-based measurements. Moreover, we 317 adjusted the analyses for somatic maturation, which is an important potential 318 confounder because of its association with metabolic risk factors, adiposity and physical 319 activity $[8,9]$.

320

\section{CONCLUSIONS}

322 In conclusion, we found that PA attenuates metabolic risk of adolescents with overweight and obesity. Although VPA seems to explain a great part of this attenuation,

324 MPA also appears important for better metabolic profile. During growth and 
development, adolescents with overweight and more physical activity show, in general,

similar metabolic risk to their counterparts with normal weight.

\section{Acknowledgements}

The authors thank all participants and funders of the original studies that contributed data to the ICAD, all ICAD collaborators, and Prof. Chris Riddoch, Prof. Ken Judge, and Dr. Pippa Griew. The ICAD collaborators include Prof. S. Anderssen, Norwegian School for Sport Science, Oslo, Norway (EYHS, Norway); Prof. G. Cardon, Department of Movement and Sports Sciences, Ghent University, Belgium (Belgium Preschool Study); Centers for Disease Control and Prevention, National Center for Health Statistics, Hyattsville, Maryland, USA (NHANES); Prof. A. Cooper, Centre for Exercise, Nutrition and Health Sciences, University of Bristol, Bristol, UK (Personal and Environmental Associations with Children's Health [PEACH]); Dr. R. Davey, Centre for Research and Action in Public Health, University of Canberra, Canberra, Australia (Children's Health and Activity Monitoring for Schools [CHAMPS]); Prof. U. Ekelund, Norwegian School of Sport Sciences, Oslo, Norway, and MRC Epidemiology Unit, University of Cambridge, Cambridge, UK; Dr. D.W. Esliger, School of Sports, Exercise, and Health Sciences, Loughborough University, Loughborough, UK; Dr. K. Froberg, University of Southern Denmark, Odense, Denmark (EYHS, Denmark); Dr. P. Hallal, Postgraduate Program in Epidemiology, Federal University of Pelotas, Pelotas, Brazil (1993 Pelotas Birth Cohort); Prof. K.F. Janz, Department of Health and Human Physiology, Department of Epidemiology, University of Iowa, Iowa City, Iowa, USA (Iowa Bone Development Study); Dr. K. Kordas, School of Social and Community Medicine, University of Bristol, Bristol, UK (Avon Longitudinal Study of Parents and Children [ALSPAC]); Dr. S. Kriemler, Institute of Social and Preventive Medicine, University of Z€urich, Z€urich, Switzerland (Kinder-Sportstudie [KISS]); Dr. A. Page, Centre for Exercise, Nutrition and Health Sciences, University of Bristol, Bristol, UK; Prof. R. Pate, Department of Exercise Science, University of South Carolina, Columbia, South Carolina, USA (Physical Activity in Preschool Children [CHAMPS-US] and Project Trial of Activity for Adolescent Girls [Project TAAG]); Dr. J.J. Puder, Service of Endocrinology, Diabetes and Metabolism, Lausanne University Hospital, University of Lausanne, Lausanne, Switzerland (Ballabeina Study); Prof. J. Reilly, Physical Activity for Health Group, School of Psychological Sciences and Health, University of Strathclyde, Glasgow, UK (Movement and Activity Glasgow Intervention in Children [MAGIC]); Prof. J. Salmon, School of Exercise and Nutrition Sciences, Deakin University, Melbourne, Australia (Children Living in Active Neigbourhoods [CLAN]); Dr. L.B. Sherar, School of Sports, Exercise and Health Sciences, Loughborough University, Loughborough, UK; Dr. A. Timperio, Centre for Physical Activity and Nutrition Research, Deakin University, Melbourne, Australia (Healthy Eating and Play Study [HEAPS]); Dr. E.M.F. van Sluijs, MRC Epidemiology Unit, University of Cambridge, Cambridge, UK (Sport, Physical activity and Eating behavior: Environmental Determinants in Young people [SPEEDY]). The UK Medical Research Council and the Wellcome Trust (Grant ref: 102215/2/13/2) and the University of Bristol provide core support for ALSPAC. Moreover, we acknowledge São Paulo Research Foundation (FAPESP) for the master's degree scholarship of AOW (FAPESP process: 2017/27234-2). 


\section{Conflict of interest}

373 The authors declare that they have no conflict of interest.

374

375

376

377

378

379

380

381

382

383

\section{REFERENCES}

1. Han JC, Lawlor DA, Kimm SY. Childhood obesity. The Lancet. 2010;375:1737-48.

2. Kuzik N, Carson V, Andersen LB, Sardinha LB, Grøntved A, Hansen BH, et al. Physical Activity and Sedentary Time Associations with Metabolic Health Across Weight Statuses in Children and Adolescents. Obesity. 2017;25:1762-9.

3. Kuschnir MCC, Bloch KV, Szklo M, Klein CH, Barufaldi LA, De Azevedo Abreu G, et al. ERICA: Prevalence of metabolic syndrome in Brazilian adolescents. Revista de Saude Publica. 2016;50:1s-13s.

4. Moore JB, Beets MW, Brazendale K, Blair SN, Pate RR, Andersen LB, et al. Associations of Vigorous-Intensity Physical Activity with Biomarkers in Youth. Medicine and Science in Sports and Exercise. 2017;49:1366-74.

5. Werneck AO, Oyeyemi AL, Gerage AM, Cyrino ES, Szwarcwald CL, Sardinha LB, et al. Does leisure-time physical activity attenuate or eliminate the positive association between obesity and high blood pressure? Journal of Clinical Hypertension. 2018;

6. Loprinzi P, Smit E, Lee H, Crespo C, Andersen R, Blair SN. The "fit but fat" paradigm addressed using accelerometer-determined physical activity data. North American journal of medical sciences. 2014;6:295-301.

7. Rubin DA, Hackney AC. Inflammatory cytokines and metabolic risk factors during growth and maturation: influence of physical activity. Medicine and sport science. 2010;55:43-55.

8. Werneck AO, Silva DR, Collings PJ, Fernandes RA, Ronque ERV, Barbosa DS, et al. Biological Maturation, Central Adiposity, and Metabolic Risk in Adolescents: A Mediation Analysis. Childhood Obesity. 2016;12.

9. Cumming SP, Sherar LB, Pindus DM, Coelho-e-Silva MJ, Malina RM, Jardine PR. A biocultural model of maturity-associated variance in adolescent physical activity. School of Health. 2012;5:37-41.

10. Sherar LB, Griew P, Esliger DW, Cooper AR, Ekelund U, Judge K, et al. International children's accelerometry database (ICAD): design and methods. BMC public health. 2011;11:485.

11. Ekelund U, Luan J, Sherar LB, Esliger DW, Griew P, Cooper A, et al. Moderate to vigorous physical activity and sedentary time and cardiometabolic risk factors in children and adolescents. JAMA. 2012;307:704-12.

12. Eisenmann JC. On the use of a continuous metabolic syndrome score in pediatric research. Cardiovascular Diabetology. 2008;7:17.

13. Andersen LB, Harro M, Sardinha LB, Froberg K, Ekelund U, Brage S, et al. Physical activity and clustered cardiovascular risk in children: a cross-sectional study (The European Youth Heart Study). 2006;368:6.

14. Cole TJ, Bellizzi MC, Flegal KM, Dietz WH. Establishing a standard definition for child overweight and obesity worldwide: international survey. BMJ (Clinical research 
ed). 2000;320:1240-3.

15. Evenson KR, Catellier DJ, Gill K, Ondrak KS, McMurray RG. Calibration of two objective measures of physical activity for children. Journal of sports sciences. 2008;26:1557-65.

16. Moore SA, McKay HA, Macdonald H, Nettlefold L, Baxter-Jones ADG, Cameron $\mathrm{N}$, et al. Enhancing a somatic maturity prediction model. Medicine and Science in Sports and Exercise. 2015;47:1755-64.

17. Gardner MJ, Altman DG. Statistics in Medicine Confidence intervals rather than $P$ values: estimation rather than hypothesis testing. British Medical Journal (Clinical research ed). 1986;292:746-750.

18. Werneck AO, Oyeyemi AL, Gerage AM, Cyrino ES, Szwarcwald CL, Sardinha LB, et al. Does leisure-time physical activity attenuate or eliminate the positive association between obesity and high blood pressure? The Journal of Clinical Hypertension. 2018;20:959-66.

19. Frohnert BI, Jacobs DR, Steinberger J, Moran A, Steffen LM, Sinaiko AR. Relation between serum free fatty acids and adiposity, insulin resistance, and cardiovascular risk factors from adolescence to adulthood. Diabetes. 2013;62:3163-9.

20. Simpson J, Smith ADAC, Fraser A, Sattar N, Lindsay RS, Ring SM, et al. Programming of adiposity in childhood and adolescence: Associations with birth weight and cord blood adipokines. Journal of Clinical Endocrinology and Metabolism. 2017;102:499-506.

21. Weiss R, Dziura J, Burgert TS, Tamborlane W V, Taksali SE, Yeckel CW, et al. Obesity and the metabolic syndrome in children and adolescents. The New England Journal of Medicine. 2004;350:2362-74.

22. Tarp J, Bugge A, Andersen LB, Sardinha LB, Ekelund U, Brage S, et al. Does adiposity mediate the relationship between physical activity and biological risk factors in youth?: a cross-sectional study from the International Children's Accelerometry Database (ICAD). International Journal of Obesity. 2017;1-8.

23. Werneck AO, Oyeyemi AL, Fernandes RA, Romanzini M, Ronque ERV, Cyrino $\mathrm{ES}$, et al. Regional socioeconomic inequalities in physical activity and sedentary behavior among brazilian adolescents. Journal of Physical Activity and Health. 2018;15. 24. Spees CK, Scott JM, Taylor CA. Differences in amounts and types of physical activity by obesity status in US adults. American journal of health behavior. 2012;36:56-65.

25. Augustijn MJCM, D'Hondt E, Van Acker L, De Guchtenaere A, Lenoir M, Caeyenberghs K, et al. Role of Motor Competence and Executive Functioning in Weight Loss. Journal of Developmental \& Behavioral Pediatrics. 2018;0:1.

26. Howard B, Winkler EAH, Sethi P, Carson V, Ridgers ND, Salmon J, et al. Associations of Low- and High-Intensity Light Activity with Cardiometabolic Biomarkers. Medicine and Science in Sports and Exercise. 2015;47:2093-101.

27. Ekblom-Bak E, Ekblom Ö, Bergström G, Börjesson M. Isotemporal substitution of sedentary time by physical activity of different intensities and bout lengths, and its associations with metabolic risk. European journal of preventive cardiology. 2016;23:967-74.

28. Martinez-Gomez D, Eisenmann JC, Gomez-Martinez S, Veses A, Romeo J, Veiga $\mathrm{OL}$, et al. Associations of physical activity and fitness with adipocytokines in adolescents: the AFINOS Study. Nutrition, metabolism, and cardiovascular diseases : NMCD. 2012;22:252-9. 
MVPA physical activity

Boys

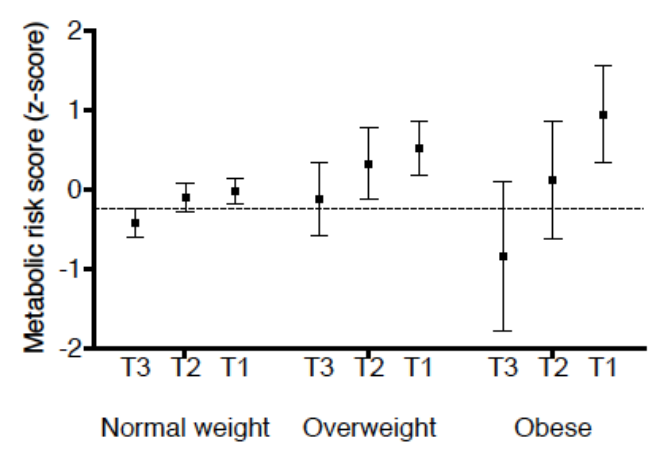

Girls

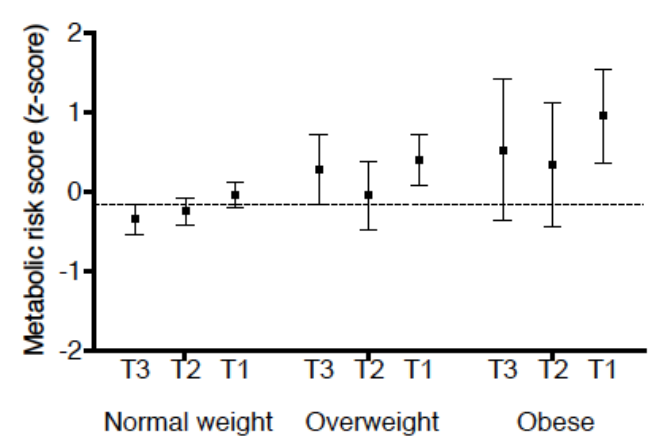

Vigorous physical activity

Boys

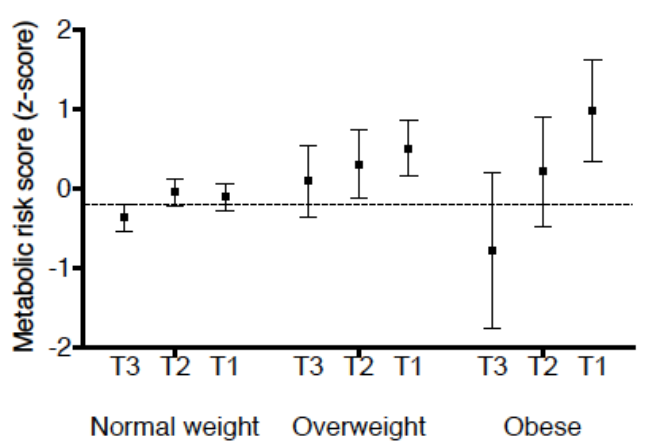

Girls

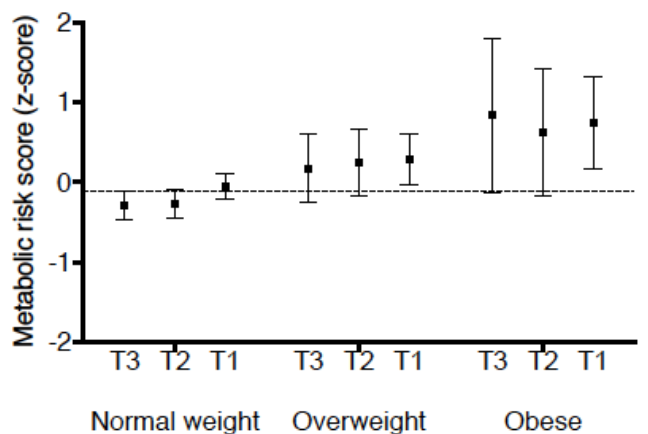

Figure 1. Metabolic risk score according to weight status and tertiles of different intensities of physical activity in adolescents.

Note. Values are presented in estimated marginal means, with $95 \%$ confidence intervals. Models were adjusted by accelerometer wear time, study, chronological age, age at peak height velocity, sedentary time and body mass index. 
Table 1. Characteristics of the included and excluded sample.

\begin{tabular}{lcc}
\hline & $\begin{array}{c}\text { Included } \\
(\mathbf{n}=\mathbf{5 , 2 1 6})\end{array}$ & $\begin{array}{c}\text { Excluded } \\
(\mathbf{n = 1 6 , 4 5 1})\end{array}$ \\
\hline Chronological age, y & $14.6(14.6$ to 14.7$)$ & $12.4(12.4$ to 12.5$)$ \\
Sex (male), \% & $48.7(47.0$ to 50.3$)$ & $47.4(46.7$ to 48.2$)$ \\
Mothers' education*, \% & $51.4(49.7$ to 53.0$)$ & $53.7(52.7$ to 54.6$)$ \\
MVPA, min/day & $44.7(43.9$ to 45.5$)$ & $51.3(50.9$ to 51.7$)$ \\
VPA, min/day & $14.5(14.1$ to 14.9$)$ & $15.5(15.3$ to 15.7$)$ \\
ST, (min/day) & $449.2(446.0$ to 452.4$)$ & $384.2(382.7$ to 385.7$)$ \\
APHV, y & $12.5(12.4$ to 12.5$)$ & $12.2(12.1$ to 12.2$)$ \\
Body mass index & & \\
Normal weight, \% & $79.5(78.4$ to 80.6$)$ & $80.8(80.2$ to 81.4$)$ \\
Overweight, \% & $14.4(13.5$ to 15.4$)$ & $5.1(4.8$ to 5.4$)$ \\
Obese, \% & $6.1(5.5$ to 6.8$)$ & (13.6 to $)$ \\
\hline Note. Values are presented in mean/frequency and $95 \%$ confidence intervals. MVPA, \\
moderate to vigorous physical activity. VPA, vigorous physical activity. ST, sedentary \\
time. APHV, age at peak height velocity. *Threshold: "any post-compulsory education \\
including vocational training", data is available for 3,497 of included participants and \\
10,726 of not-included participants.
\end{tabular}


Table 2. Characteristics of the included sample by sex $(n=5,216)$.

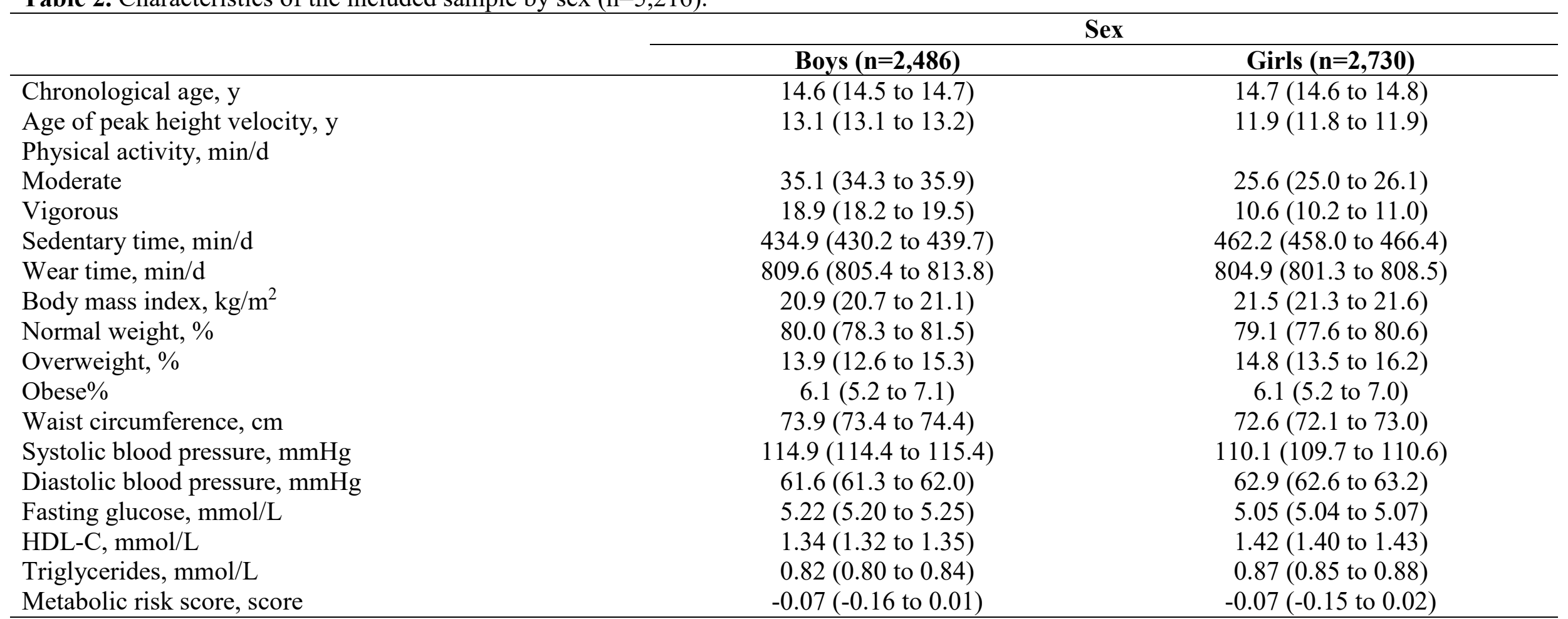

Note. Values are presented in means and 95\% confidence intervals. 
Table 3. Combined association between body mass index, different intensities of physical activity and metabolic risk among adolescents $(\mathrm{n}=5,216)$.

\begin{tabular}{|c|c|c|c|c|}
\hline & \multicolumn{2}{|l|}{ Boys } & \multicolumn{2}{|l|}{ Girls } \\
\hline & $\beta(95 \% \mathrm{CI})$ & $\mathrm{r}^{2}$ & $\beta(95 \% \mathrm{CI})$ & $\mathrm{r}^{2}$ \\
\hline Single model & & 0.177 & & 0.107 \\
\hline Body mass index & $0.215(0.194$ to 0.236$)$ & & $0.161(0.142$ to 0.181$)$ & \\
\hline Vigorous PA & & 0.180 & & 0.108 \\
\hline Body mass index & $0.213(0.191$ to 0.234$)$ & & $0.161(0.141$ to 0.180$)$ & \\
\hline Vigorous PA & $-0.009(-0.014$ to -0.003$)$ & & $-0.008(-0.016$ to -0.001$)$ & \\
\hline Moderate to vigorous PA & & 0.185 & & 0.110 \\
\hline Body mass index & $0.212(0.191$ to 0.233$)$ & & $0.161(0.141$ to 0.180$)$ & \\
\hline Moderate to vigorous PA & $-0.008(-0.011$ to -0.005$)$ & & $-0.007(-0.011$ to -0.003$)$ & \\
\hline
\end{tabular}

Note. Models were adjusted by accelerometer wear time (when including PA intensities), sedentary time, study, chronological age and age at peak height velocity. CI, confidence interval. PA, physical activity. Values in bold represents $\mathrm{p}<0.05$. 
Table 4. Regression models to the role of body mass index and moderate vigorous activity on metabolic risk according tertiles of vigorous physical activity in normal weight and overweight/obese adolescents.

\begin{tabular}{|c|c|c|}
\hline & $\beta$ & $95 \% \mathrm{CI}$ \\
\hline \multicolumn{3}{|l|}{ Normal weight } \\
\hline \multicolumn{3}{|c|}{ VPA Tertile 1} \\
\hline Body mass index & 0.166 & 0.114 to 0.217 \\
\hline Moderate PA & -0.011 & -0.021 to -0.001 \\
\hline \multicolumn{3}{|c|}{ VPA Tertile 2} \\
\hline Body mass index & 0.090 & 0.034 to 0.147 \\
\hline Moderate PA & -0.008 & -0.017 to 0.000 \\
\hline \multicolumn{3}{|c|}{ VPA Tertile 3} \\
\hline Body mass index & 0.108 & 0.052 to 0.165 \\
\hline Moderate PA & -0.013 & -0.020 to -0.006 \\
\hline \multicolumn{3}{|l|}{ Overweight/obese } \\
\hline \multicolumn{3}{|c|}{ VPA Tertile 1} \\
\hline Body mass index & 0.166 & 0.118 to 0.214 \\
\hline Moderate PA & -0.001 & -0.023 to 0.021 \\
\hline \multicolumn{3}{|c|}{ VPA Tertile 2} \\
\hline Body mass index & 0.175 & 0.101 to 0.249 \\
\hline Moderate PA & -0.027 & -0.052 to -0.003 \\
\hline \multicolumn{3}{|c|}{ VPA Tertile 3} \\
\hline Body mass index & 0.205 & 0.104 to 0.306 \\
\hline Moderate PA & -0.012 & -0.035 to 0.011 \\
\hline
\end{tabular}

Note. Models were adjusted by sex, accelerometer wear time, sedentary time, study, chronological age and age at peak height velocity. CI, confidence interval. PA, physical activity. Values in bold represents $\mathrm{p}<0.05$. 